\title{
DUBINS-RRT PATH PLANNING AND HEADING-VECTOR CONTROL GUIDANCE FOR A UUV RECOVERY
}

\author{
Zheping Yan, ${ }^{*}$ Bing Hao, ${ }^{* * *}$ Wei Zhang, ${ }^{*}$ and Simon X. Yang ${ }^{* * *}$
}

\begin{abstract}
When a UUV (unmanned underwater vehicle) completes a specific mission, it needs to return to the recovery platform autonomously safely in an unknown environment. So the works of path planning and guidance control for a UUV are indispensable and significant. In this paper, first a smooth and practical Dubins path back to the recovery platform is planned. In particular, the combined Dubins-RRT path (rapidly exploring random tree based on Dubins path) is introduced with densely spread obstacles. Then the UUV is guided along the Dubins-RRT path to the target by heading-vector control. The algorithm is simple and subjects to the kinematics constraints of UUV. The effectiveness of the algorithm is proved by the simulation in real geographical condition.
\end{abstract}

\section{Key Words}

UUV, path planning, heading-vector, guidance, recovery

\section{Introduction}

More and more unmanned underwater vehicles (UUVs) are applied to specific ocean tasks. In general, it takes hours or tens of hours for a UUV to perform a task in the ocean, longer in some other detection tasks even more. Although the UUV can emerge to the water surface to transmit data by radio, the emerging process significantly consumes the task time especially in the deep sea. The solution to this problem is that UUV autonomously return to a platform where it could exchange data and get charged and maintenance. Thus the problem of UUV endurance and information exchange can be solved.

In recent years many recovery methods and guidance strategies have been developed based on UUV platform and

* College of Automation, Harbin Engineering University, Harbin, Heilongjiang Province, China; e-mail: yanzheping@ hrbeu.edu.cn, dawizw@163.com

** College of Computer and Control Engineering, Qiqihar University, Qiqihar, Heilongjiang Province, China; e-mail: haobing_ learning@163.com

*** Advanced Robotics and Intelligent Systems Laboratory, School of Engineering, University of Guelph, Guelph, ON, Canada; e-mail: syang@uoguelph.ca

Recommended by Prof. Howard Li

(DOI: 10.2316/Journal.206.2016.3.206-4770) experiments are also carried out to validate the strategies. Paper [1] designed a sliding mode controller to regulate and track a premeditated path to complete the process of homing and docking. The design of an electromagnetic guidance system is given in [2]. This system is particularly suitable for a wide class of underwater robots. In [3], a current observer based on kinematics constraints is designed to compensate drift caused by ocean currents. The contribution of paper [4] is to give a mathematical method of calculus of variations of a recovery path for a submerged mobile. The bio-inspired control method and the cascaded controller consisting of a kinematics controller and a dynamic controller are proposed in [5]. To complete the reconfiguration of an appropriate control law, control energy cost function is used in [6] as the optimization criterion. And the particle swarm optimization algorithm is utilized to solve the control reallocation problem. Nonetheless most of the experiments show that these recovery methods are time-consuming, thus reduce the entire vehicle operation time, as well as work efficiency. To adapt more different tasks, we should improve the reliability and efficiency of the existing methods.

In general, when a UUV is about to enter the docking stage, it should keep the same heading with the recovery platform. To solve this problem, this paper proposes a planning method of rapidly exploring random tree based on Dubins path (Dubins-RRT) [7]-[10]. The Dubins algorithm was first proposed by L.E. Dubins in 1957 [11]. Dubins path algorithm tries to find the shortest path between two vectors, and this path is composed of several straight line segments and arc segments. In this paper, Dubins path is formed with the position and heading of the UUV as the starting vector and the position and heading of recovery platform as the finishing vector. Then the UUV sails along the planned Dubins path and arrives at the finishing point in a fixed direction.

When the path has been planned, the UUV needs to track the path by controlling the actuators. Tracking control can be divided into two types: one is the path following control, which designs a path that is not related to time; the other is the trajectory tracking control, with the reference trajectory being time-varying. The former is static geometric position tracking and the latter is the 
dynamic trajectory tracking. The recovery path studied in this paper is not changed with time, which belongs to the first type.

Some non-linear control technologies, including nonlinear intelligent technologies, such as the backstepping method [12], [13], fuzzy control [14], neural networks [15], [16], sliding mode control [17] and model predictive control [18], have been widely applied to the following control of UUV. However, these non-linear control methods are applied, and additional parameters and iterations are also required during the calculation. So these technologies are difficult to put practice into the low-cost navigation control systems.

This paper focuses on the low-cost UUV navigation control system by heading-vector based sliding mode controller [19], [20]. A heading-vector field is constructed to indicate the expected headings of the UUV at every position. And by following the expected headings the UUV is gradually guided to the desired path. The remainder of the paper is organized as follows. In Section 2, the conditions and background of the presented problem are explained. The generation method of Dubins-RRT path in an obstacle-rich environment is presented in Section 3. In Section 4 , the recovery path guidance in the heading-vector field is given. Simulation results are shown in Section 5.

\section{Problem Statement}

When the task is finished, UUV turns to the autonomous recovery stage and arrives at the rendezvous point after a period of navigation. First UUV detects the position and heading of the platform by sonar, and then the path planner plans the recovery path based on the current position and heading of UUV as a starting vector and the position and heading of the platform as the finishing vector (assuming the starting vector and the end vector being in the same plane). In many cases the UUV is in an unknown environment full of static or dynamic obstacles, in addition to the global planning before navigation. It must offer the corresponding obstacle-avoidance algorithm to meet the demands of the kinematics constraints.

As shown in Fig. 1, $n_{o}=\left(x_{o}, y_{o}, \alpha_{o}\right)$ and $n_{d}=\left(x_{d}, y_{d}\right.$, $\alpha_{d}$ ) represent the coordinates and azimuth angle of the starting vector and the finishing vector, respectively, then

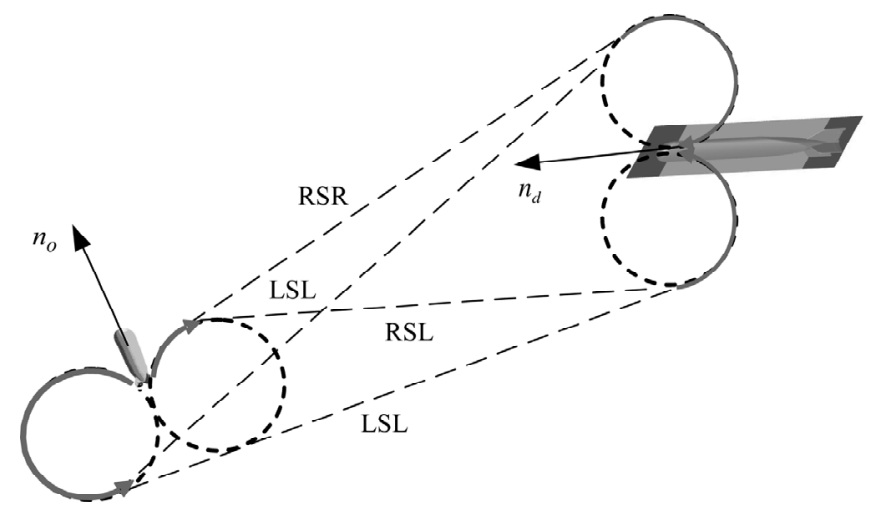

Figure 1. Four forms of Dubins path. the Dubins curve has four possible forms, namely LSL, RSR, RSL, LSR. The shortest of length is the required path. For the detailed planning algorithm, refer to [21].

\section{The Generation Method of Dubins-RRT Curve in an Obstacle-rich Environment}

If a UUV is in an obstacle-rich environment, some simple avoidance method can also be applied, but the regenerated sub-path may intersect with the obstacle repeatedly and another sub-path is required. Computer calculation time and data storage may increase significantly when multiple iterations are performed. This paper presents a Dubins path planner based on searching map of RRT in the obstacle-rich environments, which can reduce the computing time and the amount of data [22].

\subsection{Generating the Intermediate Waypoints with the Guiding Sampling Method}

Combined with Dubins curve, RRT algorithm is used to generate the waypoints in the recovery area. The sampling nodes are selected by means of the guiding sampling method, and the new sampling points and the sub-nodes in the original tree are connected by the dynamic Dubins curve. The radius of curvature of the dynamic Dubins curve is generated randomly to meet the constraint. With the growth of the tree structure, a set of Dubins curve form, the shortest of which will be selected.

The process of the Dubins curve based RRT algorithm [20] is given as follows:

1. Initialization.

2. If not reaching the target position, turn to step (3), otherwise, if the random tree having been constructed, then turn to step (7).

3 . Set $t_{\max }$ to be the maximum running time. If $t>t_{\max }$ turn to step (5), otherwise turn to step (4).

4. Generate a random node $N_{\text {rand }}$ in the search space and calculate the corresponding candidate node $N_{\text {new }}$.

5. Set $N_{\text {rand }}$ to be the finishing point and determine an $N_{\text {near }}$ according to it, then get an $N_{\text {new }}$.

6. Determine whether potential threats exist between $N_{\text {new }}$ and $N_{\text {near }}$. If no threats, the two nodes will be connected by the Dubins arc, and this $N_{\text {new }}$ is added into the random tree, then go to step (2). If threats are existing, turn to step (3).

7. Look up reversely in the feasible paths of the constructed random tree to form the final path.

To control the size of the random tree (i.e., not too deep and not too wide), this paper developed the guiding random sampling method to generate the $N_{\text {rand }}$ node, namely to design the related parameters to control the generation of the sampling point.

First of all, control RRT depth (the path length from the current node to the root node). The depth value of each node should be considered in search of the "nearest" node to the sampling node, which stimulates a shorter path to the root node and limits some sampling points' generating in the process. In this way, some fixed node to the longer path is removed and the final path is optimized. 
The distance from the current node to the sampling node is calculated by:

$$
\begin{aligned}
D= & D_{\text {staight }}\left(N, N_{\text {rand }}\right)+D_{1} * \Delta o\left(N, N_{\text {rand }}\right) \\
& +D_{2} * \operatorname{rand}(0,1) * \operatorname{depth} h_{\text {tree }}(N)
\end{aligned}
$$

where $N$ represents the current node in the growing tree; $N_{\text {rand }}$ the sampling node; $o$ the direction of the node. The construction of the tree is subjected to the straight distance from the current node to the sampling node, the direction gap between the two nodes, and the depth value from current node to the root node has to the root node. Various environments determine the design of parameters $D_{1}$ and $D_{2}$, whose values reflect the influence on the tree growth.

There are four possible connections between two vectors in traditional Dubins path. LSL, RSR, RSL, LSR. In this paper, LSL, RSR will be simplified as the coorientation Dubins path while RSL, LSR as the antiorientation Dubins path.

For the co-orientation Dubins path case, the composition of the path is shown in Fig. 2.

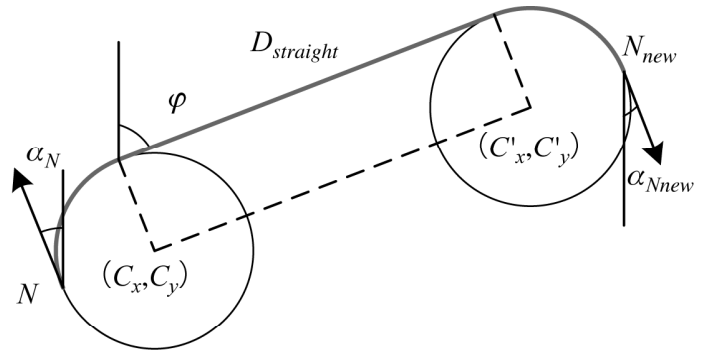

Figure 2. The composition of the co-orientation Dubins path.

The centre point coordinates of the node $N$ is defined as:

$$
\left(C_{x}, C_{y}\right)=\left(x_{N} \pm R \cos \alpha_{N}, y_{N} \pm R \sin \alpha_{N}\right)
$$

The centre point coordinates of the new generation node $N_{\text {new }}$ is defined as:

$$
\left(C_{x}^{\prime}, C_{y}^{\prime}\right)=\left(x_{N_{\text {new }}} \pm R \cos \alpha_{N_{\text {new }}}, y_{N_{\text {new }}} \pm R \sin \alpha_{N_{\text {new }}}\right)
$$

$\varphi$ is the angle between the straight path and the longitudinal axis, which can be calculated by:

$$
\varphi=\frac{\pi}{2}-\arctan \left(\frac{C_{y}^{\prime}-C_{y}}{C_{x}^{\prime}-C_{x}}\right)
$$

$D_{\text {straight }}$ is the path length of the line segment, which can be calculated by:

$$
D_{\text {straight }}=\sqrt{\left(C_{x}^{\prime}-C_{x}\right)^{2}+\left(C_{y}^{\prime}-C_{y}\right)^{2}}
$$

For the anti-orientation Dubins path case, the composition of the path is shown in Fig. 3.

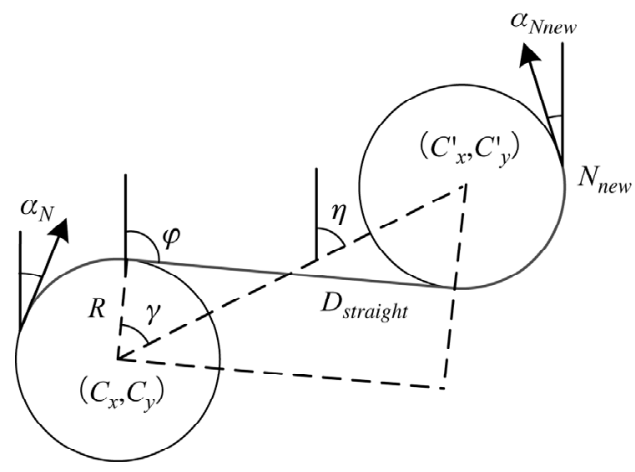

Figure 3. The composition of the anti-orientation Dubins path.

The coordinates of the centre point is the same with the co-orientation Dubins path. $\varphi$ is the angle between the straight path and the longitudinal axis. $\eta$ is the angle between the line connecting the two centre points and the longitudinal axis. $\gamma$ is the angle between the line connecting the two centre points and the radius. These angles can be calculated by:

$$
\begin{gathered}
\varphi=\eta-\gamma+\frac{\pi}{2} \\
\eta=\frac{\pi}{2}-\arctan \left(\frac{C_{y}^{\prime}-C_{y}}{C_{x}^{\prime}-C_{x}}\right) \\
\gamma=\arctan \left(\frac{2 R}{d}\right)
\end{gathered}
$$

Secondly, to limit the width of the generated tree, the product of the value of a random parameter multiplying the depth value of the previous sampling is the width constraint of the new node. By this guiding sampling method, the new sampling node will be distributed within a range of the tree.

Again, the turning angle for the new node is selected in the scope of $\left(-\frac{\pi}{2}, \frac{\pi}{2}\right)$. However, the random node sampling is hard to achieve with direction constraint. Even if such nodes are found, the path generated may lead to unnecessary turning, thus UUV may waste time and the limited energy. To solve this problem, an auxiliary observer is designed in this paper. When the new sampling node is generated, no obstacles lie between the two nodes and a Dubins path forms; this node is added to the tree as a new node, otherwise it is abandoned and the next sampling begins again. $\alpha_{N_{n e w}}$ is denoted as the direction of the new node. $K_{L}$ is the slope of the connection line between the new node and the target point (Fig. 4).

$$
\alpha_{N_{\text {new }}}=\arctan K_{L}-\left(\frac{\pi}{2}-\alpha_{N}\right)
$$

\subsection{Dynamic Dubins Curve}

Environment under the sea is mysterious and UUV cannot navigate by large turning radius in small area. A UUV 


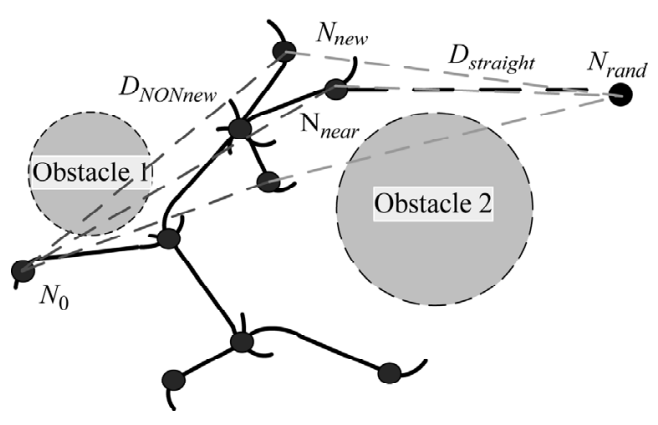

Figure 4. Expansion of Dubins-RRT with the guiding sampling.

cannot navigate by large turning radius in a small area, while in broad areas too small turning radius may cause unnecessary rotary added into the tree, which affects the effectiveness. This paper connects the adjacent nodes by dynamic Dubins curves to adapt to different geographical environment. First, a time parameter is set in the growth process of the tree. If a reasonable path cannot be found within this period of time, another turning radius will be re-selected. The method of selecting the turning radius is to increase the turning radius by a small constant in the specified range until it meets the requirements.

\section{Recovery Path Guidance by Heading-Vector Control}

In the above-mentioned work, the Dubins-RRT path from the rendezvous point of UUV to the recovery platform place is formed, and the following work is to guide UUV to the path using the navigation algorithm. The guiding method in this paper is implemented by heading-vector control. A heading-vector field is constructed to indicate the expected headings of the UUV at every position. Application of this guiding method ensures an approximate-to-zero tracking error in constant turbulence. The controller designed to follow the path is treated as the inner loop control of the whole control system of UUV, aiming at generating heading control commands for the outer loop controller to produce corresponding propeller and rudder angle commands.

Height and speed of UUV are assumed constant. The simplified kinematics model of UUV is given by:

$$
\begin{aligned}
& \dot{x}=V_{P} \sin \chi+O C_{x} \\
& \dot{y}=V_{P} \cos \chi+O C_{y}
\end{aligned}
$$

where $V_{P}$ represents the speed of UUV at $p$ in the earthfixed coordinate. $\left(O C_{x}, O C_{y}\right)$ represents two velocity components of ocean current at the point $p . \quad \chi$ is the heading of UUV at the current point. If $V_{G}$ represents the speed of UUV in the fixed coordinate, then the relationship between $V_{P}, V_{G}$ and $O C$ can be shown in Fig. 5 .

An alternative representation of (10) can be developed by:

$$
\begin{aligned}
& V_{G x}=V_{P} \sin \chi+O C_{x} \\
& V_{G y}=V_{P} \cos \chi+O C_{y}
\end{aligned}
$$

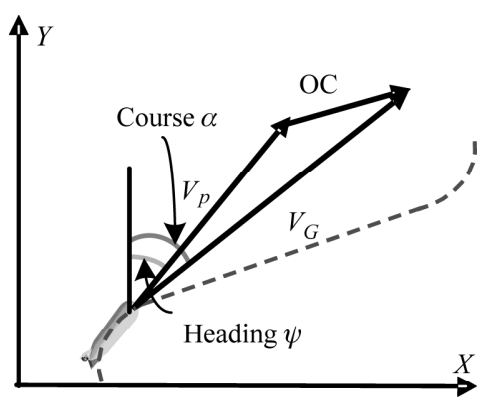

Figure 5. Relationship between $V_{P}, V_{G}$ and $O C$.

Drawing on (11) and the definition of course $\alpha$ shown in Fig. 5, (12) can be expressed as:

$$
\begin{aligned}
& \dot{x}=V_{G} \sin \alpha \\
& \dot{y}=V_{G} \cos \alpha
\end{aligned}
$$

In formula (12), the UUV heading and the speed relative to currents are replaced with the navigation direction and the navigation speed in earth-fixed coordinate system. Path following control in the vector field helps to avoid the current disturbance. This is particularly important for small UUVs.

The planned path is discrete and composed of several sub-Dubins paths. Each sub-Dubins path is composed of line segments and arc segments. So the navigation algorithm design should also be discrete, dividing into straight line guidance algorithm and arc guidance algorithm.

\subsection{Following the Straight Line by Heading-Vector Control}

As shown in Fig. 6, the thick solid line is the target path for UUV to follow, and is the centre line of the heading-vector field. The direction of each vector in vector field represents the desired course of the UUV at this point. The regulation of the desired heading-vector changes as the angle between the vector and the target line decreases, and the distance from the vector to the target path decreases.

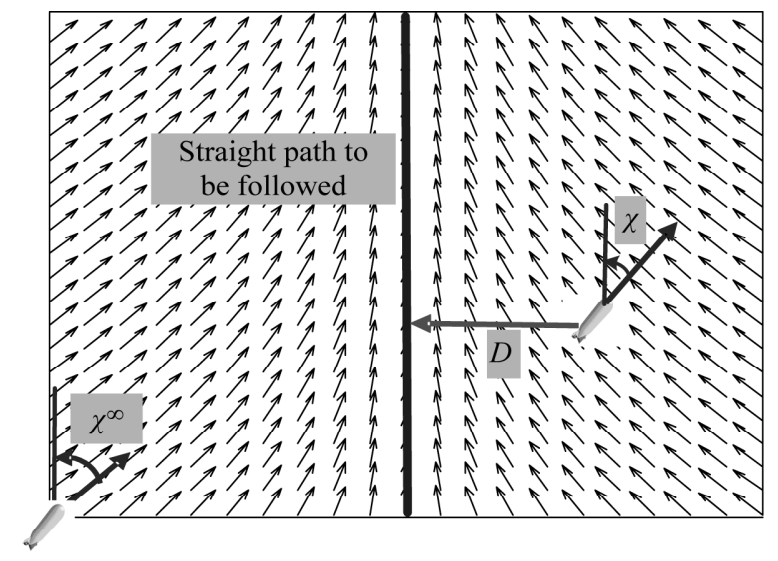

Figure 6. Following the straight line in the vector field. 
$D$ is the lateral distance from the current location of UUV to the target line. $\chi$ is the angle between the current heading of UUV and the target line. The goal is to construct a heading-vector field. When $D$ is large, UUV approaches to the target path on a fixed course and when $D$ is close to 0 , UUV sails in the same direction with the target line.

For discussion purpose, the direction of the target line is assumed to be the $Y$ axis direction, then the lateral distance from the UUV to the target line is $D=x$.

$\chi_{\max }$ represents the course angle approaching target line. The desired heading angle of the UUV is designed as:

$$
\chi_{d}(x)=-\chi_{\max } \cdot f(x)
$$

The explanation of choosing $f(x)$ is as follows.

1. $f(x)$ is strictly monotonically increasing odd function within the range of $x$ and $f(0)=0$.

2. This function is a bounded function, and its range is $(-1,1)$.

3. The derivative of $f(x)$ is a monotonically decreasing function in $(0,+\infty)$.

$f(x)=\frac{1-e^{-\beta x}}{1+e^{-\beta x}}$ is selected in this paper. $\beta$ is a positive definite parameter, which affects the changing speed of the heading angle from $\chi_{\max }$ to zero. Set $\chi_{\max } \in\left(0, \frac{\pi}{2}\right)$, and according to $f(x)$ we have $\chi_{d}(x) \in\left(-\frac{\pi}{2}, \frac{\pi}{2}\right)$.

The definition of the Lyapunov function

$$
W_{1}=\frac{1}{2} x^{2}
$$

and its derivative

$$
\dot{W}_{1}=x \dot{x}=x V_{G} \sin \chi
$$

When $\chi=\chi_{d}(x)$,

$$
\dot{W}_{1}=x V_{G} \sin \chi_{d}=-x V_{G} \sin \left(\chi_{\max } \times \frac{1-e^{-\beta x}}{1+e^{-\beta x}}\right)
$$

where $x, \sin (\cdot)$ and $\frac{1-e^{-\beta x}}{1+e^{-\beta x}}$ are all odd functions, then $\dot{W}_{1}<0$. If $\chi=\chi_{d}(x)$, then $y \rightarrow 0$.

Assume that UUV can achieve closed-loop control by on-board controller, and the designed controller is the kinematics controller of the outer loop and its equation can be defined as:

$$
\dot{\chi}=K\left(\chi_{c}-\chi\right)+v
$$

where $K, v$ are definite parameters. $K$ represents the response speed of the outer loop controller, $v$ represents unmodelled dynamics or external disturbance. $K$ and $v$ are usually difficult to be precisely predicted in experiments and the estimation error is inevitable. The adaptive sliding mode control method is adopted in this paper to fulfil the course control of UUV.
The sliding surface is chosen as:

$$
S(\chi)=e_{\chi}+K_{I} \int_{0}^{t} e_{\chi} d \tau
$$

where $K_{I}$ is the integral gain. Let $e_{\chi}=\chi-\chi_{d}(x)$, the differential of (15) can be calculated as:

$$
\dot{S}(\chi)=\dot{e}_{\chi}+K_{I} e_{\chi}=K\left(\chi_{c}-\chi\right)+v-\dot{\chi}_{d}+K_{I} e_{\chi}
$$

Select the Lyapunov function $W_{2}=\frac{1}{2} S^{2}$, take the derivative as:

$$
\dot{W}_{2}=S \dot{S}=S\left(K\left(\chi_{c}-\chi\right)+v-\dot{\chi}_{d}+K_{I} e_{\chi}\right)
$$

Then, the control signal input is selected as:

$$
\chi_{c}=\chi+\frac{1}{\hat{K}}\left(-\hat{v}+\dot{\chi}_{d}-K_{I} e_{\chi}-K_{1} S-K_{2} \operatorname{sign}(S)\right)
$$

where $\dot{\chi}_{d}$ is the differential of $\chi_{d}, \hat{K}$ and $\hat{v}$ are the estimated values of the uncertain parameters $K$ and $v, K_{1}$ and $K_{2}$ are the positive constants.

$$
\operatorname{sign}(x)= \begin{cases}1, & \text { if } x>0 \\ 0, & \text { if } x=0 \\ -1, & \text { if } x<0\end{cases}
$$

$\Delta \chi=\chi_{c}-\chi, \quad(16)$ is substituted into (17) and let $\tilde{K}=K-\hat{K}$, which can be developed by:

$$
\begin{aligned}
\dot{W}_{2} & =S\left(K \Delta \chi+v-\dot{\chi}_{d}+K_{I} e_{\chi}\right) \\
& =S\left((K-\hat{K}) \Delta \chi+\hat{K} \Delta \chi+v-\dot{\chi}_{d}+K_{I} e_{\chi}\right) \\
& =S\left(\tilde{K} \Delta \chi+\hat{K}\left(\chi_{c}-\chi\right)+v-\dot{\chi}_{d}+K_{I} e_{\chi}\right)
\end{aligned}
$$

Substitute (18) into (19), and let $\tilde{v}=v-\hat{v}$, which can be developed by:

$$
\begin{aligned}
\dot{W}_{2} & =S\left(\tilde{K} \Delta \chi+\hat{K}\left(\chi_{c}-\chi\right)+v-\dot{\chi}_{d}+K_{I} e_{\chi}\right) \\
& =S\left(\tilde{K} \Delta \chi-\hat{v}+v-K_{1} S-K_{2} \operatorname{sign}(S)\right) \\
& =S\left(\tilde{K} \Delta \chi+\tilde{v}-K_{1} S-K_{2} \operatorname{sign}(S)\right)
\end{aligned}
$$

To obtain the estimate values $\hat{K}$ and $\hat{v}$, Lyapunov function $W_{3}$ is defined as:

$$
W_{3}=\frac{1}{2} S^{2}+\frac{1}{2} \rho_{v}^{-1} \tilde{v}^{2}+\frac{1}{2} \rho_{K}^{-1} \tilde{K}^{2}
$$

$\rho_{v}, \rho_{K}$ are the positive parameters, the differential of (21) can be calculated as:

$$
\begin{aligned}
\dot{W}_{3} & =S\left(\tilde{K} \Delta \chi+\tilde{v}-K_{1} S-K_{2} \operatorname{sign}(S)\right)+\rho_{v}^{-1} \tilde{v} \dot{\tilde{v}}+\rho_{K}^{-1} \tilde{K} \dot{\tilde{K}} \\
& =-K_{1} S^{2}-K_{2}|S|+\tilde{v}\left(S-\rho_{v}^{-1} \dot{\tilde{v}}\right)+\tilde{K}\left(S \Delta \chi-\rho_{K}^{-1} \dot{\tilde{K}}\right)
\end{aligned}
$$


As $K$ and $v$ are constant values, then $\dot{\tilde{K}}=\dot{\hat{K}}, \dot{\tilde{v}}=\dot{\hat{v}}$, are substituted into (22). Equation (22) is updated as:

$\dot{W}_{3}=-K_{1} S^{2}-K_{2}|S|+\tilde{v}\left(\mathrm{~S}-\rho_{v}^{-1} \dot{\hat{v}}\right)+\tilde{K}\left(S \Delta \chi-\rho_{K}^{-1} \dot{\hat{K}}\right)$

The adaptation law for $\hat{K}$ and $\hat{v}$ can be:

$$
\begin{gathered}
\dot{\hat{K}}=\rho_{K} S \Delta \chi \\
\dot{\hat{v}}=\rho_{v} S
\end{gathered}
$$

So $\dot{W}_{3}=-K_{1} S^{2}-K_{2}|S| \leq 0$ is obtained. The sliding surface $S$ can approach to zero in a finite time, $e_{\chi}$ will converge to zero too.

To eliminate chattering effect caused by a discontinuous sign function, the control signal input can be replaced by:

$$
\chi_{c}=\chi+\frac{1}{\hat{K}}\left(-\hat{v}+\dot{\chi}_{d}-K_{I} e_{\chi}-K_{3} s a t\left(\frac{S}{\varepsilon}\right)\right)
$$

where $\operatorname{sat}(x)= \begin{cases}1, & \text { if }|x| \leq 1 \\ \operatorname{sign}(x), & \text { otherwise }\end{cases}$

In addition, a border region around the sliding surface is defined as $S_{\varepsilon}=\{|S| \leq \varepsilon\}$.

As the sliding surface is a progressive convergence, when $|S| \geq \varepsilon, S$ can reach into $S_{\varepsilon}$ within a finite time. Therefore, region $S_{\varepsilon}$ is the only case considered for the stability proof.

Theorem 1. The equations of the system are given by (12) and (16), the controlled heading and the desired heading of UUV can be given in (26) and (13). If the maximum of the heading angle is in the range of $\left(0, \frac{\pi}{2}\right]$, and $\frac{\chi_{\max } \beta}{\mu} \min \left(\frac{\bar{x}}{\varepsilon}, \frac{K_{3} \theta}{\varepsilon V_{G}}\right)>1$, where

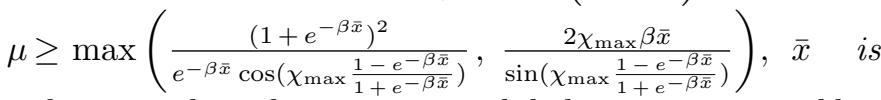
arbitrary, then this system is global asymptotic stable.

Proof: Lyapunov function is defined as:

$$
W=\frac{1}{2} x^{2}+\frac{1}{2} \theta S^{2}
$$

Differentiating, we obtain

$$
\begin{aligned}
\dot{W} & =x \dot{x}+\theta S \dot{S}=x V_{G} \sin \chi+\theta S\left(\dot{e}_{\chi}+K_{I} e_{\chi}\right) \\
& =x V_{G} \sin \chi+\theta S\left(\dot{\chi}-\dot{\chi}_{d}+K_{I} e_{\chi}\right) \\
& =x V_{G} \sin \chi+\theta S\left(\hat{K}\left(\chi_{c}-\chi\right)+\hat{v}-\dot{\chi}_{d}+K_{I} e_{\chi}\right)
\end{aligned}
$$

If $\left|\frac{S}{\varepsilon}\right| \leq 1$ in the border region $S_{\varepsilon}$, the control signal is a saturated function.

$$
\chi_{c}=\chi+\frac{1}{\hat{K}}\left(-\hat{v}+\dot{\chi}_{d}-K_{I} e_{\chi}-K_{3} \frac{S}{\varepsilon}\right)
$$

So the differential of $W$ is updated as:

$$
\begin{aligned}
\dot{W}= & x V_{G} \sin \left(\chi_{d}(x)+e_{\chi}\right)-\theta \frac{K_{3}}{\varepsilon} S^{2} \\
= & -\theta \frac{K_{3}}{\varepsilon} S^{2}+x V_{G} \sin \left(\chi_{d}(x)\right) \\
& +x V_{G}\left(\sin \left(\chi_{d}(x)+e_{\chi}\right)-\sin \left(\chi_{d}(x)\right)\right. \\
\leq & -\theta \frac{K_{3}}{\varepsilon} S^{2}+x V_{G} \sin \left(\chi_{d}(x)\right) \\
& +|x| V_{G} \mid \sin \left(\chi_{d}(x)+e_{\chi}\right)-\sin \left(\chi_{d}(x) \mid\right.
\end{aligned}
$$

Let:

$$
\begin{aligned}
\delta & =\mid \sin \left(\chi_{d}(x)+e_{\chi}\right)-\sin \left(\chi_{d}(x) \mid\right. \\
& =\mid \sin \left(\chi_{d}(x)\right) \cos e_{\chi}+\cos \left(\chi_{d}(x)\right) \sin e_{\chi}-\sin \left(\chi_{d}(x) \mid\right. \\
& =\left|\sin \left(\chi_{d}(x)\right)\left(\cos e_{\chi}-1\right)+\cos \left(\chi_{d}(x)\right) \sin e_{\chi}\right| \\
& \leq\left|\cos e_{\chi}-1\right|+\left|\sin e_{\chi}\right| \leq 2\left|e_{\chi}\right| \leq 2|S|
\end{aligned}
$$

We get:

$$
\begin{aligned}
\dot{W} & \leq-\theta \frac{K_{3}}{\varepsilon} S^{2}+x V_{G} \sin \left(\chi_{d}(x)\right)+2 V_{G}|x||S| \\
& =-\theta \frac{K_{3}}{\varepsilon} S^{2}+2 V_{G}|x||S|-x V_{G} \sin \left(\chi_{\max } \frac{1-e^{-\beta x}}{1+e^{-\beta x}}\right)
\end{aligned}
$$

Let:

$$
\xi(x)=x \sin \left(\chi_{\max } \frac{1-e^{-\beta x}}{1+e^{-\beta x}}\right)
$$

To find the conditions of $\dot{W} \leq 0$, even function $\zeta(x)$ is constructed in the range $(-\infty, \infty)$, which ensures $\xi(0)=\zeta(0)=0$ and $0 \leq \xi(x) \leq \zeta(x)$. Consider the function:

$$
\zeta(x)=\left\{\begin{array}{ll}
\frac{\chi_{\max } \beta}{\mu} x^{2}, & \text { if }|x| \leq \bar{x} \\
\frac{\chi_{\max } \beta}{\mu} \bar{x}(2|x|-\bar{x}), & \text { if }|x|>\bar{x}
\end{array}, \quad \bar{x}\right. \text { is arbitrary }
$$

As both $\xi(x)$ and $\zeta(x)$ are even functions, $x \geq 0$ is the only case considered in the following.

When $0 \leq x \leq \bar{x}$,

$$
\begin{aligned}
& \xi^{\prime}(x)= \sin \left(\chi_{\max } \frac{1-e^{-\beta x}}{1+e^{-\beta x}}\right) \\
&+x \chi_{\max } \cos \left(\chi_{\max } \frac{1-e^{-\beta x}}{1+e^{-\beta x}}\right) \frac{2 \beta e^{-\beta x}}{\left(1+e^{-\beta x}\right)^{2}} \\
& \geq x \chi_{\max } \cos \left(\chi_{\max } \frac{1-e^{-\beta \bar{x}}}{1+e^{-\beta \bar{x}}}\right) \frac{2 \beta e^{-\beta \bar{x}}}{\left(1+e^{-\beta \bar{x}}\right)^{2}} \\
& \zeta^{\prime}(x)=\frac{2 x \chi_{\max } \beta}{\mu}
\end{aligned}
$$

If $\zeta^{\prime}(x) \leq \xi^{\prime}(x)$ and $\xi(0)=\zeta(0)=0$, then $\zeta(x) \leq \xi(x)$

$$
\frac{2 x \chi_{\max } \beta}{\mu} \leq x \chi_{\max } \cos \left(\chi_{\max } \frac{1-e^{-\beta \bar{x}}}{1+e^{-\beta \bar{x}}}\right) \frac{2 \beta e^{-\beta \bar{x}}}{\left(1+e^{-\beta \bar{x}}\right)^{2}}
$$


And then, $\mu \geq \frac{\left(1+e^{-\beta \bar{x}}\right)^{2}}{e^{-\beta \bar{x}} \cos \left(\chi_{\max } \frac{1-e^{-\beta \bar{x}}}{1+e^{-\beta \bar{x}}}\right)}$

When $x>\bar{x}$,

$$
\begin{aligned}
& \xi(x)=x \sin \left(\chi_{\max } \frac{1-e^{-\beta x}}{1+e^{-\beta x}}\right) \geq x \sin \left(\chi_{\max } \frac{1-e^{-\beta \bar{x}}}{1+e^{-\beta \bar{x}}}\right) \\
& \zeta(x)=\frac{\chi_{\max } \beta}{\mu} \bar{x}(2|x|-\bar{x}) \leq 2 \frac{\chi_{\max } \beta}{\mu} \bar{x} \cdot x
\end{aligned}
$$

Let $\zeta(x) \leq \xi(x)$, that is:

$$
2 \frac{\chi_{\max } \beta}{\mu} \bar{x} \cdot x \leq x \sin \left(\chi_{\max } \frac{1-e^{-\beta \bar{x}}}{1+e^{-\beta \bar{x}}}\right)
$$

which is equivalent to:

$$
\mu \geq \frac{2 \chi_{\max } \beta \bar{x}}{\sin \left(\chi_{\max } \frac{1-e^{-\beta \bar{x}}}{1+e^{-\beta \bar{x}}}\right)}
$$

$0 \leq x \leq \bar{x}$ and $\quad x>\bar{x}$ are integrated, if $\mu \geq$ $\max \left(\frac{\left(1+e^{-\beta \bar{x}}\right)^{2}}{e^{-\beta \bar{x}} \cos \left(\chi_{\max } \frac{1-e^{-\beta \bar{x}}}{1+e^{-\beta \bar{x}}}\right)}, \frac{2 \chi_{\max } \beta \bar{x}}{\sin \left(\chi_{\max } \frac{1-e^{-\beta \bar{x}}}{1+e^{-\beta \bar{x}}}\right)}\right)$, then $\zeta(x) \leq$ $\xi(x)$, which implies that:

$$
\dot{W} \leq-\theta \frac{K_{3}}{\varepsilon} S^{2}+2 V_{G}|x||S|-V_{G} \zeta(x)
$$

Therefore, when $0 \leq x \leq \bar{x}$, we get:

$$
\begin{aligned}
\dot{W} & \leq-\frac{K_{3} \theta}{\varepsilon} S^{2}+2 V_{G}|x||S|-V_{G} \frac{\chi_{\max } \beta}{\mu} x^{2} \\
& =-V_{G}\left(\frac{K_{3} \theta}{\varepsilon V_{G}} S^{2}-2|x||S|+\frac{\chi_{\max } \beta}{\mu} x^{2}\right) \\
& =-V_{G}(|S||x|)\left(\begin{array}{cc}
\frac{K_{3} \theta}{\varepsilon V_{G}} & -1 \\
-1 & \frac{\chi_{\max } \beta}{\mu}
\end{array}\right)\left(\begin{array}{c}
|S| \\
|x|
\end{array}\right)
\end{aligned}
$$

If $\frac{K_{3} \theta}{\varepsilon V_{G}}\left(\frac{\chi_{\max } \beta}{\mu}\right)>1$, then $\dot{W}<0$.

When $x>\bar{x}$ and $|S| \leq \varepsilon$, we get:

$$
\begin{aligned}
\dot{W} & \leq-\frac{K_{3} \theta}{\varepsilon} S^{2}+2 V_{G}|x||S|-V_{G} \frac{\chi_{\max } \beta}{\mu} \bar{x}(2|x|-\bar{x}) \\
& \leq 2 V_{G}|x| \cdot \varepsilon-2 V_{G} \frac{\chi_{\max } \beta}{\mu} \bar{x} \cdot|x| \\
& =2 V_{G}|x|\left(\varepsilon-\frac{\chi_{\max } \beta}{\mu} \bar{x}\right)
\end{aligned}
$$

If $\frac{\chi_{\max } \beta \bar{x}}{\mu \varepsilon}>1$ then $\dot{W}<0$.

In summary, Theorem 1 is achieved.

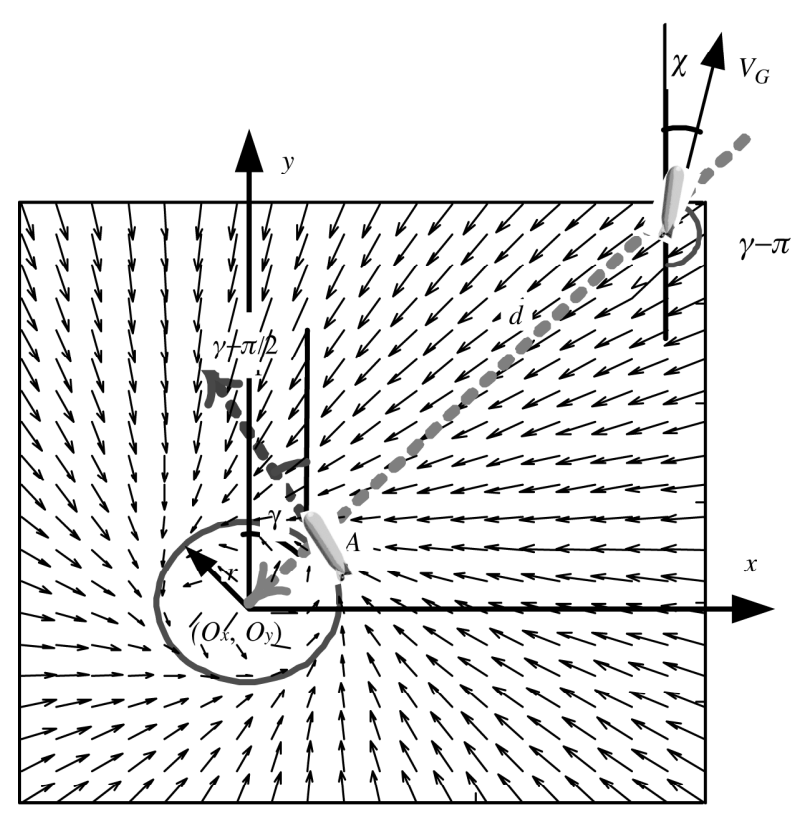

Figure 7. Following the arc segment in the vector field.

\subsection{Following the Arc Segment by Heading-Vector Control}

Following the arc segment is similar to following the straight line segments in the heading-vector field. The geometric relationship of UUV and the target arc is shown in Fig. 7. The planning path in this paper is Dubins curve in which the directions of arcs can be clockwise or counterclockwise. The counter clockwise target arc segment is discussed here and the research methodology of the clockwise arc segment is similar to it, except that the signs are different.

Assume that the radius of the target arc segment is $r$, and the coordinates of the centre point $\left(O_{x}, O_{y}\right)$ is known. The distance from UUV to the arc centre point being long, the desired navigation direction of UUV is the direction connecting UUV and the centre point. $d$ represents the distance from UUV to this centre point. The desired heading of UUV is calculated by:

$$
\chi_{d}=\gamma-\pi
$$

When $d$ gradually reduces until it is equal to $r$, the desired navigation direction of UUV is the tangential direction of the point $A$. $A$ is the point of intersection of the target arc and the line connecting UUV with the centre point. The desired heading of UUV is calculated by:

$$
\chi_{d}=\gamma-\pi / 2
$$

The desired course of UUV can be given by:

$$
\chi_{d}(\Delta d)=\gamma-\frac{\pi}{2}-\frac{\pi}{2} \frac{1-e^{-\beta \Delta d}}{1+e^{-\beta \Delta d}}
$$

$$
\Delta d=d-r
$$


In the case of the arc segment, it will bring great convenience to the problems being studied to convert the twodimensional coordinate into the polar coordinate. From Fig. 7, the coordinates of UUV can be calculated by:

$$
\begin{aligned}
& x=O_{x}+d \sin \gamma \\
& y=O_{y}+d \cos \gamma
\end{aligned}
$$

Substituted by (12) and its derivative is formed as:

$$
\begin{aligned}
& \dot{d}=V_{G} \sin (\chi-\gamma) \\
& \dot{\gamma}=\frac{V_{G}}{d} \cos (\chi-\gamma)
\end{aligned}
$$

where $V_{G}$ and $\chi$ are velocity and heading angle of UUV relative to the earth-fixed coordinates, respectively. Dynamic equation of heading is also given as (14).

The definition of the Lyapunov function:

$$
W_{1}=\frac{1}{2} \Delta d^{2}
$$

and its derivative:

$$
\dot{W}_{1}=\Delta d \Delta \dot{d}=\Delta d V_{G} \sin (\chi-\gamma)
$$

When $\chi=\chi_{d}$,

$$
\begin{aligned}
\dot{W}_{1} & =\Delta d \Delta \dot{d} \\
& =\Delta d V_{G} \sin (\chi-\gamma) \\
& =\Delta d V_{G} \sin \left(\gamma-\frac{\pi}{2}-\frac{\pi}{2} \frac{1-e^{-\beta \Delta d}}{1+e^{-\beta \Delta d}}-\gamma\right) \\
& =-\Delta d V_{G} \sin \left(\frac{\pi}{2} \frac{1-e^{-\beta \Delta d}}{1+e^{-\beta \Delta d}}\right)
\end{aligned}
$$

$\sin (\cdot)$ is monotonically increasing in $(-\pi / 2, \pi / 2), \Delta d$ is monotonically increasing too, so $\dot{W}_{1}<0$. If $\chi=\chi_{d}(\Delta d)$, $\Delta d \rightarrow 0$ asymptotically.

The design of adaptive sliding mode controller is elaborated as follows.

The sliding surface is chosen as:

$$
S(\chi)=e_{\chi}+K_{I} \int_{0}^{t} e_{\chi} d \tau
$$

where $K_{I}$ is the integral gain. Let $e_{\chi}=\chi-\chi_{d}(x)$, the differential of the sliding surface equation can be calculated as:

$$
\begin{gathered}
\dot{S}(\chi)=\dot{e}_{\chi}+K_{I} e_{\chi}=K\left(\chi_{c}-\chi\right)+v-\dot{\chi}_{d}+K_{I} e_{\chi} \\
\chi_{c}=\chi+\frac{1}{\hat{K}}\left(-\hat{v}+\dot{\chi}_{d}-K_{I} e_{\chi}-K_{3} \operatorname{sat}\left(\frac{S}{\varepsilon}\right)\right)
\end{gathered}
$$

The proof of the reachability of the sliding mode surface and the producing process of the adaptive law is similar to that in Section 4.1, so it is not repeated here.

Theorem 2. The equations of the system are given by (32) and (34), and the controlled heading and the desired heading of $U U V$ can be given in (35) and (29). If the maximum of the heading angle is in the range of $\left(0, \frac{\pi}{2}\right]$, and $\frac{\beta \pi}{\mu \varepsilon}\left(\bar{d}, \frac{K_{3} \theta}{2 V_{G}}\right)>1$, where $\mu \geq \max \left(\frac{\pi\left(1+e^{-\beta \Delta d}\right)^{2}}{2 e^{-\beta \Delta d} \cos \left(\frac{\pi}{2} \frac{1-e^{-\beta \Delta d}}{1+e^{-\beta \Delta d}}\right)}, \frac{\beta \pi \bar{d}}{2 \sin \left(\frac{\pi}{2} \frac{1-e^{-\beta \bar{d}}}{1+e^{-\beta d}}\right)}\right), \bar{d}$ is arbitrary then this system is global asymptotic stable.

Proof: Lyapunov function is defined as:

$$
W=\frac{1}{2} \Delta d^{2}+\frac{1}{2} \theta S^{2}
$$

Differentiating, we obtain:

$$
\begin{aligned}
\dot{W} & =\Delta d \Delta \dot{d}+\theta S \dot{S} \\
& =\Delta d V_{G} \sin (\chi-\gamma)+\theta S\left(\dot{e}_{\chi}+K_{I} e_{\chi}\right) \\
& =\Delta d V_{G} \sin (\chi-\gamma)+\theta S\left(\dot{\chi}-\dot{\chi}_{d}+K_{I} e_{\chi}\right) \\
& =\Delta d V_{G} \sin (\chi-\gamma)+\theta S\left(\hat{K}\left(\chi_{c}-\chi\right)+\hat{v}-\dot{\chi}_{d}+K_{I} e_{\chi}\right)
\end{aligned}
$$

If $\left|\frac{S}{\varepsilon}\right| \leq 1$ in the border region $S_{\varepsilon}$, the control signal is a saturated function.

$$
\chi_{c}=\chi+\frac{1}{\hat{K}}\left(-\hat{v}+\dot{\chi}_{d}-K_{I} e_{\chi}-K_{3} \frac{S}{\varepsilon}\right)
$$

So the differential of $W$ is updated as:

$$
\dot{W}=\Delta d V_{G} \sin \left(\chi_{d}(\Delta d)+e_{\chi}-\gamma\right)-\theta \frac{K_{3}}{\varepsilon} S^{2}
$$

Let $\hat{\chi}_{d}=\chi_{d}(\Delta d)-\gamma$, we get

$$
\begin{aligned}
\dot{W}= & -\theta \frac{K_{3}}{\varepsilon} S^{2}+\Delta d V_{G} \sin \left(\hat{\chi}_{d}(\Delta d)\right) \\
& +\Delta d V_{G}\left(\sin \left(\hat{\chi}_{d}(\Delta d)+e_{\chi}\right)-\sin \left(\hat{\chi}_{d}(\Delta d)\right)\right. \\
\leq & -\theta \frac{K_{3}}{\varepsilon} S^{2}+2 V_{G}|S||\Delta d|+\Delta d V_{G} \sin \left(\hat{\chi}_{d}(\Delta d)\right) \\
= & -\theta \frac{K_{3}}{\varepsilon} S^{2}+2 V_{G}|S||\Delta d|-\Delta d V_{G} \sin \left(\frac{\pi}{2} \frac{1-e^{-\beta \Delta d}}{1+e^{-\beta \Delta d}}\right)
\end{aligned}
$$

Let $\xi(\Delta d)=\sin \left(\frac{\pi}{2} \frac{1-e^{-\beta \Delta d}}{1+e^{-\beta \Delta d}}\right)$

To find the conditions of $\dot{W} \leq 0$, even function $\zeta(\Delta d)$ is constructed in the range $(-\infty, \infty)$, and ensures $\xi(0)=\zeta(0)=0$ and $0 \leq \xi(\Delta d) \leq \zeta(\Delta d)$. Consider the function:

$\zeta(\Delta d)=\left\{\begin{array}{l}\frac{\pi \beta}{2 \mu} \Delta d^{2}, \quad \text { if }|\Delta d| \leq \bar{d} \\ \frac{\pi \beta}{2 \mu} \bar{d}(2|\Delta d|-\bar{d}), \quad \text { if }|\Delta d|>\bar{d}\end{array}, \bar{d}\right.$ is arbitrary. 
As both $\xi(\Delta d)$ and $\zeta(\Delta d)$ are even functions, $\Delta d \geq 0$ is the only case considered in the following.

When $\quad 0 \leq \Delta d \leq \bar{d}, \quad \xi^{\prime}(\Delta d)=\Delta d \cos \left(\frac{\pi}{2} \frac{1-e^{-\beta \Delta d}}{1+e^{-\beta \Delta d}}\right)$ $\frac{2 \beta e^{-\beta \Delta d}}{\left(1+e^{-\beta \Delta d}\right)^{2}}, \zeta^{\prime}(\Delta d)=\frac{\beta \pi}{\mu} \Delta d$

Let $\zeta^{\prime}(x) \leq \xi^{\prime}(x)$, then $\frac{\beta \pi}{\mu} \Delta d \leq \Delta d \cos \left(\frac{\pi}{2} \frac{1-e^{-\beta \Delta d}}{1+e^{-\beta \Delta d}}\right)$ $2 \beta e^{-\beta \Delta d}$

$\overline{\left(1+e^{-\beta \Delta d}\right)^{2}}$.

And then, if $\mu \geq \frac{\pi\left(1+e^{-\beta \Delta d}\right)^{2}}{2 e^{-\beta \Delta d} \cos \left(\frac{\pi}{2} \frac{1-e^{-\beta \Delta d}}{1+e^{-\beta \Delta d}}\right)}$, which

implies that

$$
\begin{aligned}
\dot{W} & \leq-\frac{K_{3} \theta}{\varepsilon} S^{2}+2 V_{G}|\Delta d||S|-V_{G} \frac{\beta \pi}{2 \mu} \Delta d^{2} \\
& =-V_{G}\left(\frac{K_{3} \theta}{\varepsilon V_{G}} S^{2}-2|\Delta d||S|+\frac{\beta \pi}{2 \mu} \Delta d^{2}\right) \\
& =-V_{G}(|S||\Delta d|)\left(\begin{array}{cc}
\frac{K_{3} \theta}{\varepsilon V_{G}} & -1 \\
-1 & \frac{\beta \pi}{2 \mu}
\end{array}\right)\left(\begin{array}{c}
|S| \\
|\Delta d|
\end{array}\right)
\end{aligned}
$$

If $\frac{\beta \pi}{2 \mu}\left(\frac{K_{3} \theta}{\varepsilon V_{G}}\right)>1$, then $\dot{W}<0$.

$$
\begin{aligned}
& \text { When } \quad \Delta d>\bar{d}, \quad \xi(\Delta d)=\Delta d \sin \left(\frac{\pi}{2} \frac{1-e^{-\beta \Delta d}}{1+e^{-\beta \Delta d}}\right) \geq \\
& \Delta d \sin \left(\frac{\pi}{2} \frac{1-e^{-\beta \bar{d}}}{1+e^{-\beta \bar{d}}}\right), \zeta(\Delta d) \leq \xi(\Delta d) \\
& \zeta(\Delta d)=\frac{\beta \pi}{2 \mu} \bar{d}(2|\Delta d|-\bar{d}) \leq \frac{\beta \pi}{2 \mu} \bar{d} \cdot \Delta d \leq \Delta d \sin \left(\frac{\pi}{2} \frac{1-e^{-\beta \bar{d}}}{1+e^{-\beta \bar{d}}}\right)
\end{aligned}
$$

which is equivalent to:

$$
\mu \geq \frac{\beta \pi \bar{d}}{2 \sin \left(\frac{\pi}{2} \frac{1-e^{-\beta \bar{d}}}{1+e^{-\beta \bar{d}}}\right)}
$$

We get:

$$
\begin{aligned}
\dot{W} & \leq-\frac{K_{3} \theta}{\varepsilon} S^{2}+2 V_{G}|\Delta d||S|-V_{G} \frac{\beta \pi}{2 \mu} \bar{d}(2|\Delta d|-\bar{d}) \\
& \leq 2 V_{G}|\Delta d| \cdot \varepsilon-2 V_{G} \frac{\beta \pi}{\mu} \bar{d} \cdot|\Delta d| \\
& =2 V_{G}|\Delta d|\left(\varepsilon-\frac{\beta \pi}{\mu} \bar{d}\right)
\end{aligned}
$$

If $\frac{\beta \pi \bar{d}}{\mu \varepsilon}>1$, then $\dot{W}<0$.

Theorem 2 can be obtained by integrating the above two cases $0 \leq \Delta d \leq \bar{d}$ and $\Delta d>\bar{d}$.

\subsection{Following the Combinatorial Path of Line Seg- ments and Arc Segments}

The planned path in this paper is the combination of line segments and arc line paths, so it is required to explain how to construct the heading-vector field around the mixed path. To avoid dead zones and singular points generated in the mixed vector field, the UUV constructs the headingvector field according to the path type (line or arc) it is following. Before starting the next sub-path, the UUV is not to completely switch the heading-vector field until it arrives at the end of a present sub-path (line or arc segment). When following the mixed path in the headingvector field, the time point for conversion of different vector fields is to be specified. The methodology in this paper is to define a dynamic threshold, which is determined by the lengths of the paths and the current speed of the UUV. When the distance from UUV to the end of the present path is less than or equal to the threshold, the conversion of the heading-vector field will start.

\section{Simulation}

The simulations are carried out digitally with complex marine environment: $400 \mathrm{~m} \times 400 \mathrm{~m}$ area in the digital ocean map containing some collision areas made up of multiple islands. In the process of recovery, UUV has to detour among these islands. The vector of the rendezvous point is $(60,60, \pi)$. The vector of the position of the recovery platform is $(175,300,3 \pi / 4)$. Assume that the longitudinal velocity of UUV is $1 \mathrm{~m} / \mathrm{s}$.

Case 1: The recovery path by means of Dubins-RRT without guiding sampling

To find out the approximate optimal path, we set 4 cycles in the simulation, and select the shortest path of all the cycles as the final result. Running the program, the length of the planned path is calculated as $348.5965 \mathrm{~m}$ (Fig. 8).

Case 2: The recovery path by means of Dubins-RRT with guiding sampling

Equation (1) is used to calculate the distance from the current node to the sampling node. The parameters $D_{1}$ and $D_{2}$ of formula (1) are set as 0.1 and 0.3 , respectively. This choice of parameters is to make the direction gap between the two nodes is less effect on the distance $D$, and the depth value from current node to the root node has

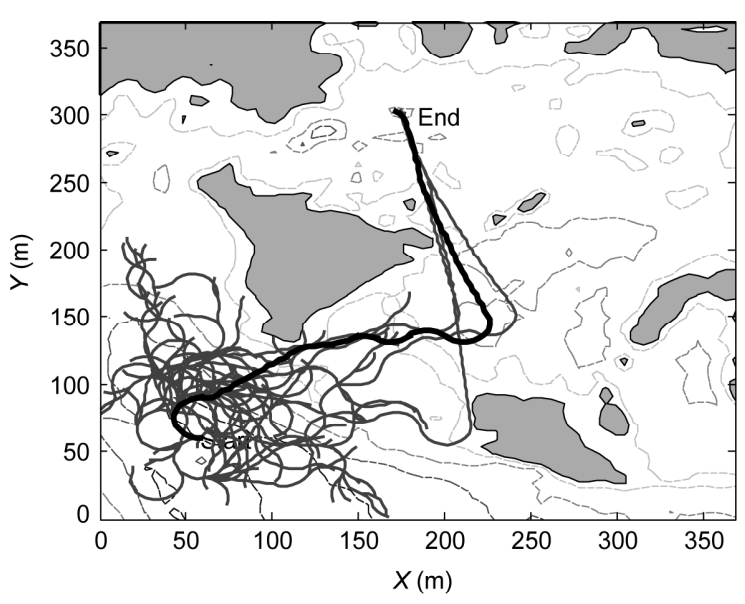

Figure 8. The path planning simulation of case 1. 


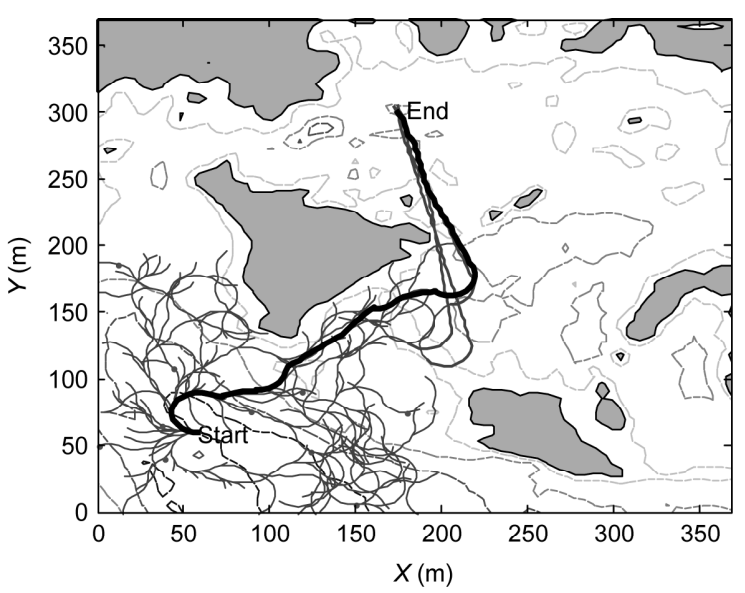

(a)

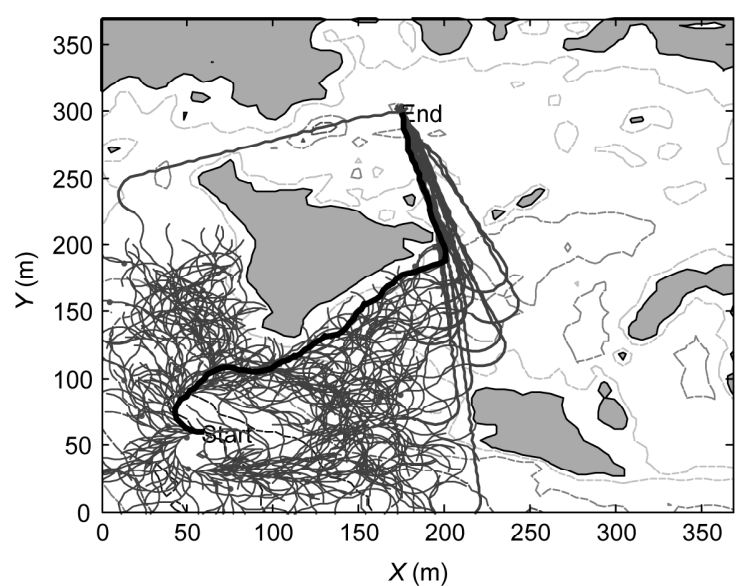

(b)

Figure 9. The path planning simulation of case 2: (a) the number of the simulation cycle is 4 and (b) the number of the simulation cycle is 20 .

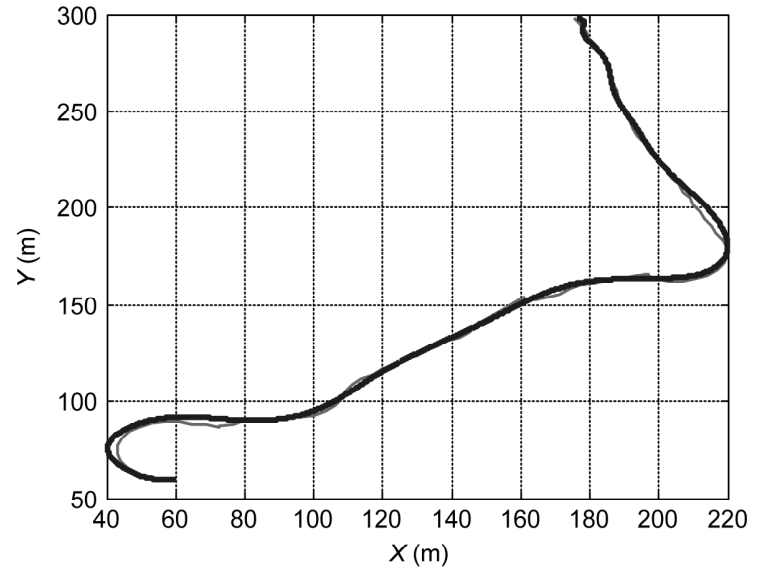

(a)

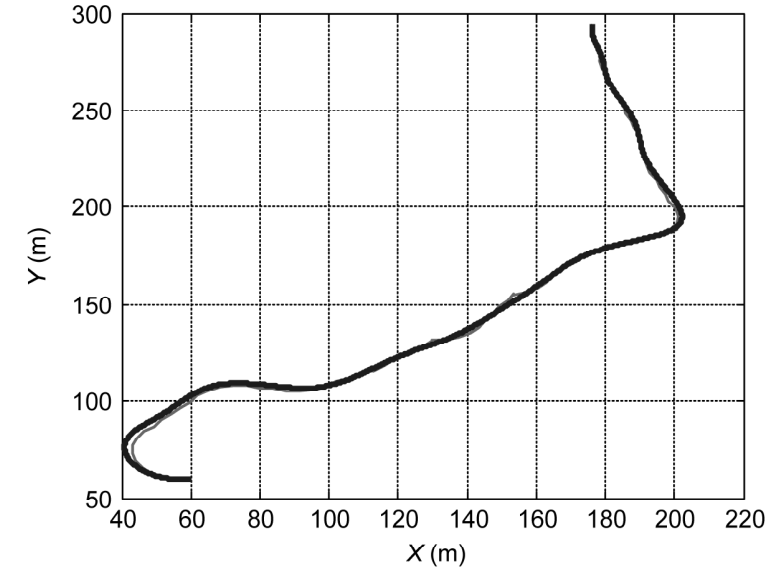

(b)

Figure 10. The path following simulation of case 2: (a) the number of the simulation cycle is 4 and (b) the number of the simulation cycle is 20 .

Table 1

Control Parameter Values

\begin{tabular}{|l|l|c|l|}
\hline $\begin{array}{l}\text { Parameter Value of the } \\
\text { Straight Path Followed }\end{array}$ & Origin & $\begin{array}{c}\text { Parameters Value of the } \\
\text { Arc Path Followed }\end{array}$ & Origin \\
\hline$\beta=12$ & Equation (13) & $\beta=20$ & Equation (29) \\
\hline$\mu=25$ & Theorem 1 & $\mu=40$ & Theorem 2 \\
\hline$K_{3}=2$ & Equation $(26)$ & $K_{3}=5$ & Equation (35) \\
\hline $\bar{x}=1$ & Theorem 1 & $\varepsilon=1$ & Theorem 2 \\
\hline$\varepsilon=2$ & Theorem 1 & $\theta=5$ & Theorem 2 \\
\hline$\theta=3$ & Theorem 1 & & \\
\hline
\end{tabular}

a great influence on the distance $D$. When the number of simulation cycles is set as 4 and 20, respectively, the path length is calculated as 340.5354 and $336.6099 \mathrm{~m}$, respectively. The two simulations in Fig. 9 show that if regardless of the recovery time, the increase in the number of simulation cycles can further optimize the path.
Figure 10 shows the results of the heading-vector field algorithm. In these simulations, the parameters of the straight path followed are set as the course angle approaching target line is set as $\chi_{\max }=\frac{\pi}{4} \cdot \chi_{\max }=\frac{\pi}{4}$, speed of UUV is set as $V_{G}=2 \mathrm{~m} / \mathrm{s}$. The other parameters which satisfy the conditions of Theorems 1 and 2 are selected as Table 1. 
As is shown, the thin line represents the planned recovery path and the thick line represents the tracking path. In the whole process, the mean values of the following errors are less than $0.5 \mathrm{~m}$, which can meet the requirements in practical applications. The following errors on the straight-line segments of the path are smaller compared with those on the arc line segments of the path. Although the connection position of adjacent line segments and arc segments shows some lateral follow errors, the length of the tracking path and the planned recovery path is similar.

\section{Conclusion}

In this paper Dubins curve is applied for a UUV to plan the obstacle-avoidance path from the rendezvous point to the recovery platform. The methods can work in real time and require no accurate UUV dynamic model. The construction of the heading-vector fields for the line segments and arc segments of a Dubins path are presented. Both theoretical proofs and simulation analysis show that the UUV can be well guided onto the planned path by heading-vector control.

\section{Acknowledgement}

This work is partially supported by the National Natural Science Foundation of China (Grant No. 51179038), the National Science Foundation for Distinguished Young Scholars of China (Grant No. 51309067) and the Natural Science Foundation of Heilongjiang Province of China (Grant No. F2015025).

\section{References}

[1] K.H. Oh, J.Y. Kim, I.W. Park, et al., A study on the control of AUV's homing and docking, IEEE Conf. on Mechatronics and Machine Vision in Practice, Chiang Mai, Thailand, 2002.

[2] M.D. Feezor, F.Y. Sorrell, P.R. Blankinship, et al., Autonomous underwater vehicle homing/docking via electromagnetic guidance, IEEE Journal of Oceanic Engineering, 26(4), 2001, $515-521$.

[3] J.Y. Park, B.H. Jun, P.M. Lee, et al., Modified linear terminal guidance for docking and a time-varying ocean current observer, 2011 IEEE Symposium on Underwater Technology (UT) and 2011 Workshop on Scientific Use of Submarine Cables and Related Technologies (SSC), IEEE, 2011, 1-6.

[4] O. Yakimenko, D.P. Horner, and D.G. Pratt Jr., AUV rendezvous trajectories generation for underwater recovery, 2008 16th Mediterranean Conf. on Control and Automation, IEEE, 2008, 1192-1197.

[5] B. Sun, D. Zhu, and S.X. Yang, A bio-inspired cascaded approach for three-dimensional tracking control of unmanned underwater vehicles, International Journal of Robotics and Automation, 29(4), 2014, 349-358.

[6] D. Zhu, J. Liu, and S.X. Yang, Particle swarm optimization approach to thruster fault-tolerant control of unmanned underwater vehicles, International Journal of Robotics and Automation, 26(3), 2011, 282.

[7] M. Shanmugavel, A. Tsourdos, B. White, et al., Co-operative path planning of multiple UAVs using Dubins paths with clothoid arcs, Control Engineering Practice, 18(9), 2010, 1084-1092.

[8] C. Hanson, J. Richardson, and A. Girard, Path planning of a Dubins vehicle for sequential target observation with ranged sensors, Proc. 2011 American Control Conf., San Francisco, CA, 2011, 1698-1703.
[9] D.J. Grymin and A.L. Crassidis, Simplified model development and trajectory determination for a UAV using the Dubins set, Proc. 2009 AIAA Atmospheric Flight Mechanics Conf., Chicago, IL, 2009, 2009-6050.

[10] S. Jeyaraman, et al., Formal techniques for the modelling and validation of a co-operating UAV team that uses Dubins set for path planning, Proc. 2005 American Control Conf., Portland, OR, 2005, 4690-4695.

[11] L.E. Dubins, On the curves of minimal length with a constraint on average curvature, and with prescribed initial and terminal positions and tangents, American Journal of Mathematics, 79(3), 1957, 497-516.

[12] L. Lapierre and D. Soetanto, Nonlinear path following control of an AUV, Ocean Engineering, 34(11-12), 2007, 1734-1744.

[13] L. Lapierre, D. Soetanto, and A. Pascoal, Nonlinear path following with applications to the control of autonomous underwater vehicles, Proc. 42nd IEEE Conf. on Decision and Control, Maui, HI, 2003, 1256-1261.

[14] S.N. Mugdha and N.S. Sahjendra, State-dependent Riccati equation-based robust dive plane control of AUV with control constraints, Ocean Engineering, 34(11), Kyoto, Japan, 2007, 1711-1723.

[15] R. Amin, A.A. Khayyat, and K.G. Osgouie, Neural networks modeling of autonomous underwater vehicle, 2010 IEEE/ASME International Conf. on Mechatronics and Embedded Systems and Applications (MESA), Kyoto, Japan, 2010, $14-19$.

[16] I. Astrov and A. Pedai, Multirate depth control of an AUV by neural network predictive controller for enhanced situational awareness, ISCIII 2011 5th International Symposium on Computational Intelligence and Intelligent Informatics, Floriana, 2011, 47-52.

[17] K. Macek, R. Philippsen, and R. Siegwart, Path following for autonomous vehicle navigation with inherent safety and dynamics margin, Intelligent Vehicles Symposium, 6, June 4-6 2008, 108-113.

[18] S.-R. Oh and J. Sun, Path following of under-actuated marine surface vessels using line-of-sight based model predictive control, Ocean Engineering, 37(2-3), 2010, 289-295.

[19] D.R. Nelson, D.B. Barber, T.W. Mclain, et al., Vector field path following for miniature air vehicles, IEEE Transactions on Robotics, 23(3), 2007, 519-529.

[20] V.M. Gonçalves, L.C.A. Pimenta, C. Maia, et al., Vector fields for robot navigation along time-varying curves in-dimensions, IEEE Transactions on Robotics, 26(4), 2010, 647-659.

[21] A.P. Aguiar, L. Cremean, and J.P. Hespanha, Position tracking for a nonlinear underactuated hovercraft: Controller design and experimental results, Proc. 42nd IEEE Conf. on Decision and Control, IEEE, 2003, 3858-3863.

[22] S. LaValle, Rapidly-exploring random trees: A New Tool for path planning, Technical Report 98-11, Computer Science Department, Iowa State University, 1998.

\section{Biographies}

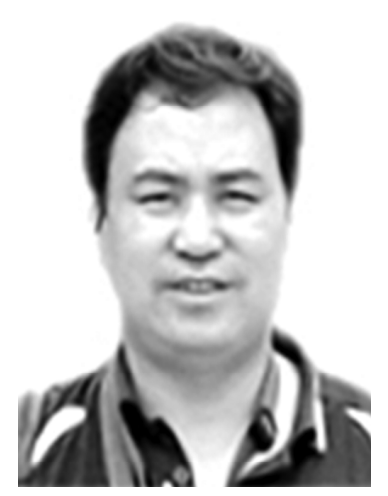

Zheping Yan was born in 1972. He finished his B.S., M.S. and Ph.D. degree in Harbin Engineering University. He finished his postdoctoral research at Harbin Institute of Technology in 2004. And he began to work in school of power and nuclear energy of Harbin Engineering University after he got the master degree. Now he is a professor and a Ph.D. supervisor of control theory and control engineering at College of Automation, Harbin Engineering University. His research interests include identification of non-linear system, multi-sensors data fusion and intelligent control. The main research directions are 
dynamic positioning system for ships, intelligent control of an autonomous underwater vehicle and deep submergence rescue vehicle.

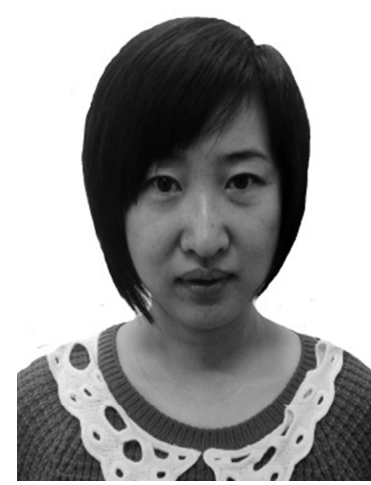

Bing Hao received a B.S. degree in automation from Qiqihar University, Qiqihar, China, in 2001, and M.S. degrees of control theory and control engineering from Qiqihar University in 2010. She has been working in College of Computer and Control Engineering, Qiqihar University, Qiqihar, China, in 2001, after he got the B.S. degree. And now she is currently working towards the Ph.D. degree in the College of Automation, Harbin Engineering University. Her current research interests include system design, path following control and depth control of an autonomous underwater vehicle and intelligent control.

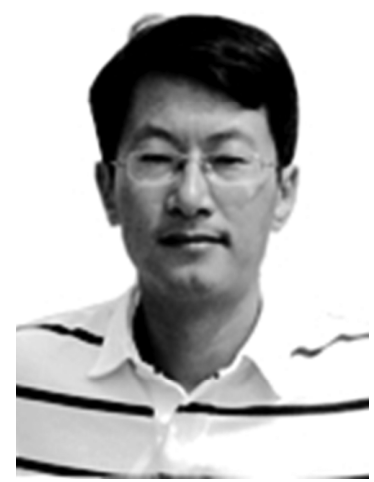

Wei Zhang was born in 1978, doctor of engineering. $\mathrm{He}$ is a Ph.D. associate supervisor and an associate professor at College of Automation, Harbin Engineering University. He finished his B.S. degree, M.S. degree and Ph.D. degree in Harbin Engineering University, respectively. After he finished his Ph.D. degree, he worked in College of Automation in Harbin Engineering University until now. His research interests include the general design of underwater unmanned vehicle (UUV), the mathematical modelling of UUV, intelligent control, data fusion, model predictive control and the docking control of UUV. Now, he assumed one item of the National Natural Science Foundation of China and one item of the Fundamental Research Funds for the Central Universities.

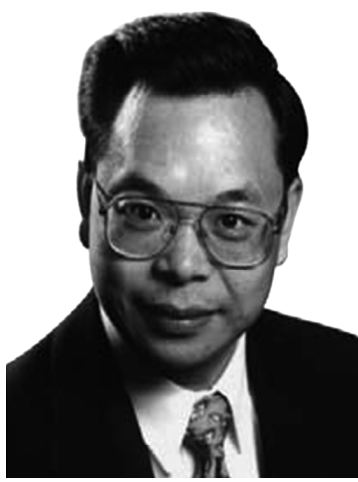

Simon $X$. Yang received the B.Sc. degree in engineering physics from Beijing University, China, in 1987, the first of two M.Sc. degrees in biophysics from the Chinese Academy of Sciences, Beijing, China, in 1990, the second M.Sc. degree in electrical engineering from the University of Houston, USA, in 1996, and the Ph.D. degree in electrical and computer engineering from the University of Alberta, Edmonton, Canada, in 1999. He joined the School of Engineering at the University of Guelph, Canada, in 1999. Currently he is a professor and the head of the Advanced Robotics and Intelligent Systems (ARIS) Laboratory at the University of Guelph, Canada. His research interests include intelligent systems, robotics, sensors and multi-sensor fusion, wireless sensor networks, control systems, soft computing and computational neuroscience. He has served as an associate editor of the IEEE Transactions on Cybernetics, the International Journal of Robotics and Automation and several other journals. He has been involved in the organization of many conferences and many other professional activities. He was the general chair of 2011 IEEE International Conference on Automation and Logistics. 